\title{
Surveillance of Gonococcal Infections with Assessment of Their Antibiotic Susceptibility Pattern
}

\author{
Rajdev Sangita ${ }^{*}$, Shah Arpita², Patel Aarohi' ${ }^{3}$, Mulla Summaiya1 \\ ${ }^{1}$ Government Medical College, Surat, India \\ ${ }^{2}$ Government Medical College, Vadodara, India \\ ${ }^{3}$ Desai metropolis laboratories, Surat, India \\ Email: “drsangitarajdev@gmail.com,arpitashah_mbbs@yahoo.co.in, aarohi.s.patel@gmail.com, \\ mullasummaiya@gmail.com
}

Received 1 April 2016; accepted 5 June 2016; published 8 June 2016

Copyright (C) 2016 by authors and Scientific Research Publishing Inc. This work is licensed under the Creative Commons Attribution International License (CC BY). http://creativecommons.org/licenses/by/4.0/

(c) (i) Open Access

\begin{abstract}
Context: Gonococcal infections are prevalent throughout the world and remain a serious public health problem, with an estimated 106.1 million new infections per year. Over recent years, the gonococcus has rapidly acquired resistance to commonly used antibiotics and very few treatment options remain; hence, regular monitoring of antimicrobial resistance is very important. Methods and Material: The study was conducted from January 2012 to March 2013 after approved by IRB \& HREC in patients attending O\&G and Skin \& VD with the complaints of vaginal discharge, cervical discharge, urethral discharge, dyspareunia, burning micturition, lower abdominal pain \& infertility. Endocervical swabs and urethral swabs were collected and cultured on chocolate agar and modified Thayer martin medium identification of organism was done by biochemical tests and antimicrobial susceptibility testing was done by Kirby-Bauer disk diffusion method as per CLSI guidelines. Results: Five Neisseria were isolated in male $(2.3 \%)$ patients of reproductive age group (20 - 30 years) with one found reactive for HIV. All patients had history of heterosexual contact of 10 - 12 days before onset of urethral discharge and burning micturition. All isolates were resistant to Penicillin, Tetracycline and Ciprofloxacin. Only one isolate was resistance to Azithromycin. Conclusions: There is a development of resistance in gonococcal isolates to third generation cephalosporin and azithromycin and these are the drugs that are given for syndromic management of cases as per NACO guidelines. So surveillance for antimicrobial resistance is crucial for monitoring the emergence and spread of antibiotic resistance in gonococcal isolates.
\end{abstract}

\footnotetext{
${ }^{*}$ Corresponding author.
} 


\section{Keywords}

\section{Neisseria gonorrhoeae, Drug Resistance}

\section{Introduction}

Gonorrhoea remains one of the most common sexually transmitted infections (STI) in developing countries and is a global health problem [1]. It is a major public health concern globally causing substantial morbidity and economic burden [2]. The prevalence of gonococcal infection varies greatly among countries in the developed and developing world, the highest being in South and Southeast Asia, followed by sub-Saharan Africa and America. According to the World Health Organization, there are approximately 62 million new cases of gonorrhoea worldwide annually, and almost half of the cases are estimated to occur in Southeast Asia [3] [4]. High rate of prevalence of gonorrhoea is due to presence of a large reservoir of asymptomatic carriers in the community and development of antimicrobial resistance to $N$. gonorrhoea [1] [5].

In the absence of any effective vaccine against $N$. gonorrhoea, control of gonococcal infection mainly depends on safe sex practices and early identification and treatment of infected individuals. Strategies for the control of gonorrhoea have relied on the use of highly-effective and often single-dose therapy administered at the time of diagnosis [3]. Therapeutic options for gonococcal infection are nowadays limited due to the spread of gonococci resistance to a wide variety of antimicrobials. Since the failure to cure a case of gonorrhoea has public health implications due to the potential for continued transmission and rapid emergence of antimicrobial resistance and facilitating the spread of HIV, sentinel surveillance and close monitoring of the in vitro antimicrobial susceptibility of clinical isolates of $N$. gonorrhoea have a crucial role in preventing the spread of resistant strains [5] [6].

Neisseria gonorrhoeae has developed resistance against antimicrobial agents such as penicillin, tetracycline and quinolones in several countries including India [2]. Antibiotic sensitivity pattern for N. gonorrhoea in different part of the world is also not uniform, and the emergence of resistance to the commonly used drugs, such as penicillin, tetracycline and ciprofloxacin is frequent in different part of the world including South East Asian Region (SEAR) [4]. During 2009-12, the percentage of penicillinase producing N. gonorrhoeae (PPNG) isolates from WHO. South East Asian Region (SEAR) varied from 0\% (Pune, Nagpur) to 88.9\% (Bhutan), while the rates of resistance to tetracycline and resistance to ciprofloxacin varied from $9.8 \%-100 \%$ and $57 \%$ - $100 \%$, respectively [2]. Therefore, continuous surveillance of antimicrobial resistance of $N$. gonorrhoea is essential to monitor its emergence and spread and to provide a rational basis for formulating national treatment guidelines [7]. So the present study was carried out to find out prevalence of gonococcal infection at our institute and study antimicrobial susceptibility pattern of Neisseria gonorrhoea isolates.

\section{Subjects and Methods}

Study population: The study was carried out from January 2012 to March 2013 in the Department of Microbiology after ethical approval from IRB in conjunction with department of Obst-Gynec \& Skin-VD. The study population comprised males with urethritis, females with complain of vaginal/cervical discharge, urethral discharge, burning micturition, lower abdominal pain, infertility and dyspareunia. All patients were included in the study after taking informed consent.

Specimen processing: Frank discharge \& or vaginal and endocervical swabs were inoculated immediately after collection on to blood agar, chocolate agar, Thayer martin medium and the inoculated plates were transported to the laboratory in candle jar for further incubation in $3 \%-7 \% \mathrm{CO}_{2}$ (candle jar) at $37^{\circ} \mathrm{C}$ for 48 hours. The colonies suspected to be $N$. gonorrhoeae were presumptively identified by Gram stain, oxidase test and superoxol test. Confirmation of identity was based on rapid carbohydrate utilisation test (RCUT). Gonococcal isolates were stored at $-70^{\circ} \mathrm{C}$ in glycerol broths [2].

Antimicrobial susceptibility testing: All isolates were examined for susceptibility to penicillin (10 IU), ciprofloxacin $(5 \mu \mathrm{g})$, tetracycline $(30 \mu \mathrm{g})$, ceftriaxone $(30 \mu \mathrm{g})$, azithromycin $(15 \mu \mathrm{g})$ by Kirby Bauer disc diffusion method as per CLSI guidelines and protocol (2012) as per shown in Picture 1. Data were expressed as percentages. 

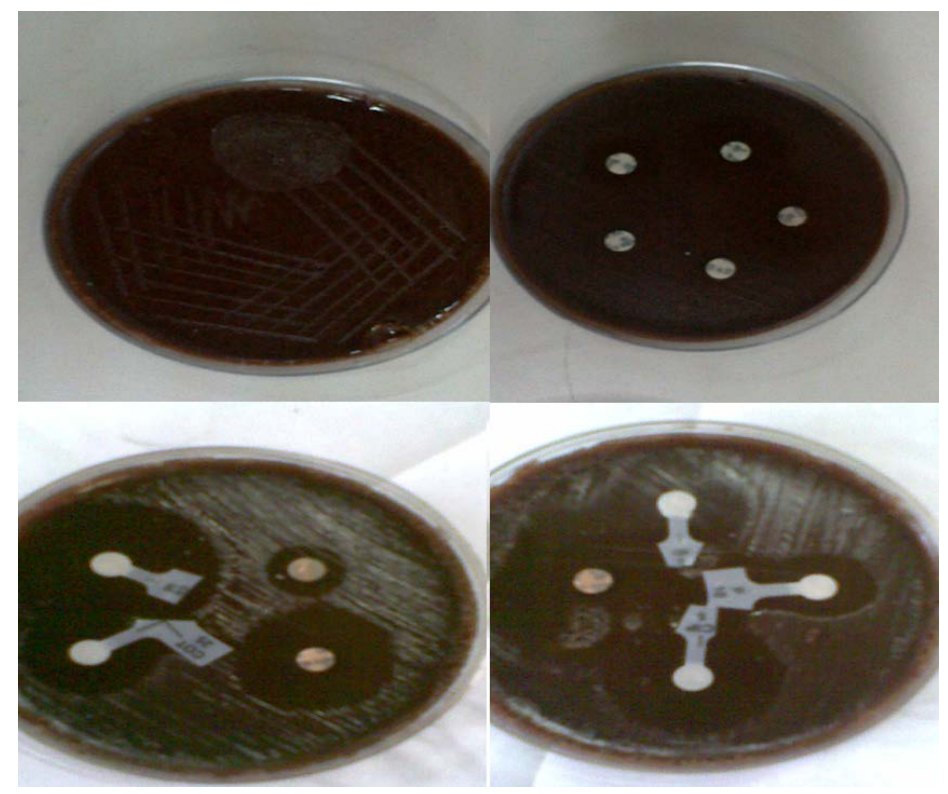

Picture 1. Culture and susceptibility.

\section{Results}

210 samples were collected from patients attending Obst and Gynaecology department \& Skin and Venereal diseases department fulfilling inclusion criteria of the study. Specimens were collected from 10 male and 200 female patients. The age of patients were ranging from 18 - 50 years (mean age 30.8 years). Maximum numbers of the patients were in the age group of 20 - 30 years (57.14\%). In present study 5 Neisseria gonorrhoea were isolated. Prevalence of gonococci isolates and age and gender wise distribution of patients in the study is as shown in Figure 1 and Figure 2 respectively. They all were positive for gram's stain in direct smear from urethral discharge. All isolates were from male patients of the age group 20 - 30 years. The isolation rate of gonococcal infection in present study was $2.3 \%$. Incidence of gonorrhoea in male patients was $50 \%$. Among five patients, one patient was reactive for HIV. Antibiotic susceptibility pattern showed that all isolates were resistant to Penicillin, Tetracycline and Ciprofloxacin. One isolate was resistant to azithromycin and two were resistant to ceftriaxone. \% sensitivity to the drugs tested is shown in Figure 3.

\section{Discussion}

According to the World Health Organization, the prevalence of sexually transmitted diseases in developing countries is about twenty times more than developed countries [8] [9]. Gonorrhoea is one of the old but re-emerging diseases in developing countries [3]. The isolation of these bacteria from patients suspected to sexually transmitted diseases varies between $4.6 \%$ to $64.7 \%$ in the world, while in India it is between $3.9 \%$ to $28 \%$ [8]. Although the main stay of management of discharge syndromes is the syndromic approach, gradual shift to the etiologic approach for diagnosis of gonorrhoea is observed. Isolation of Neisseria gonorrhoea was subsequently decreased from 2005-2011 in male patients, with respect to Khakhi et al. (87\%), Shilpee et al. (84.61\%) and Bala et al. (76.8\%). Isolation of gonococci in female patients subsequently decreased as in Khakhi et al. (18.2\%), Shilpee et al. (15.38\%) and Bala et al. (0.8\%) [2] [7] [9]-[11].

Majority of the culture-positive patients belonged to age group 20 - 30 years, which was similar with the study that Tarrah et al. and Bokaeian M et al. had reported most of the positive cases in both genders between 26 to 35 years [8] [9]. Thus the finding of present study correlates with the other studies. The possible reason for higher isolation in this age group may be sexually active population in this age groups and unsafe sex practice might be higher.

Antimicrobial resistance in Neisseria gonorrhoeae is a major public health concern worldwide. It may lead to severe complications if left untreated such as chronic pelvic inflammatory disease, ectopic pregnancy and infertility. The lack of diagnostic facilities and availability of syndromic management leads to poor isolation of 


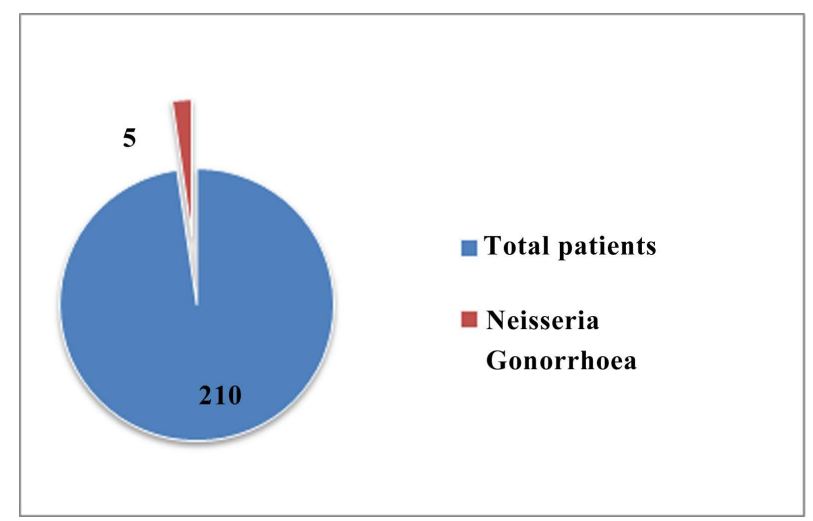

Figure 1. Prevalance of gonococcal infecions among STIs patients in numbers.

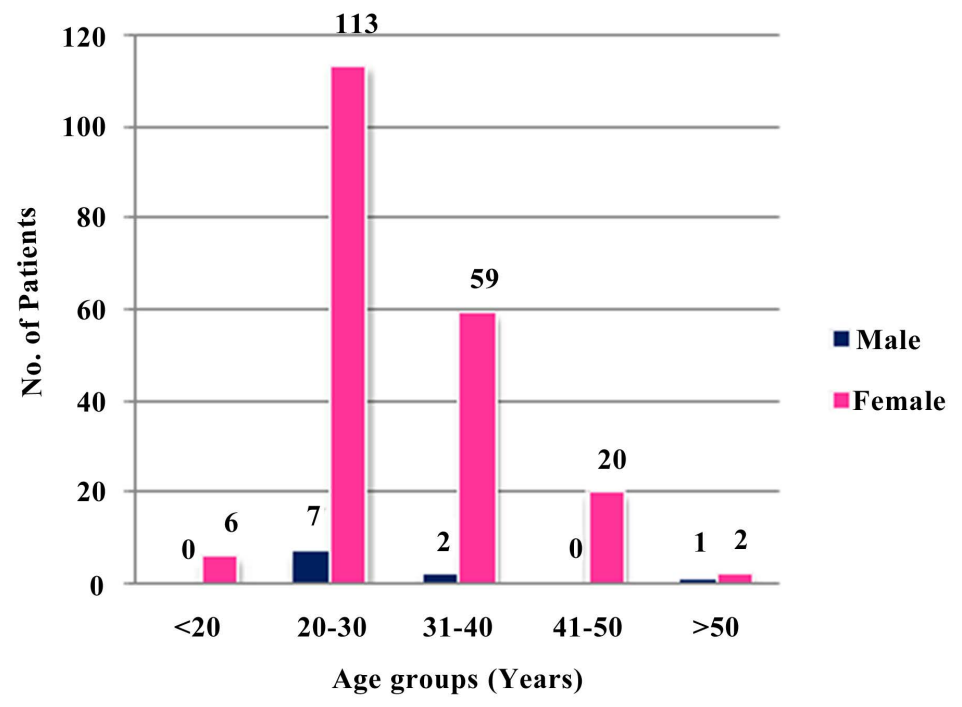

Figure 2. Age and gender wise distribution of patients.

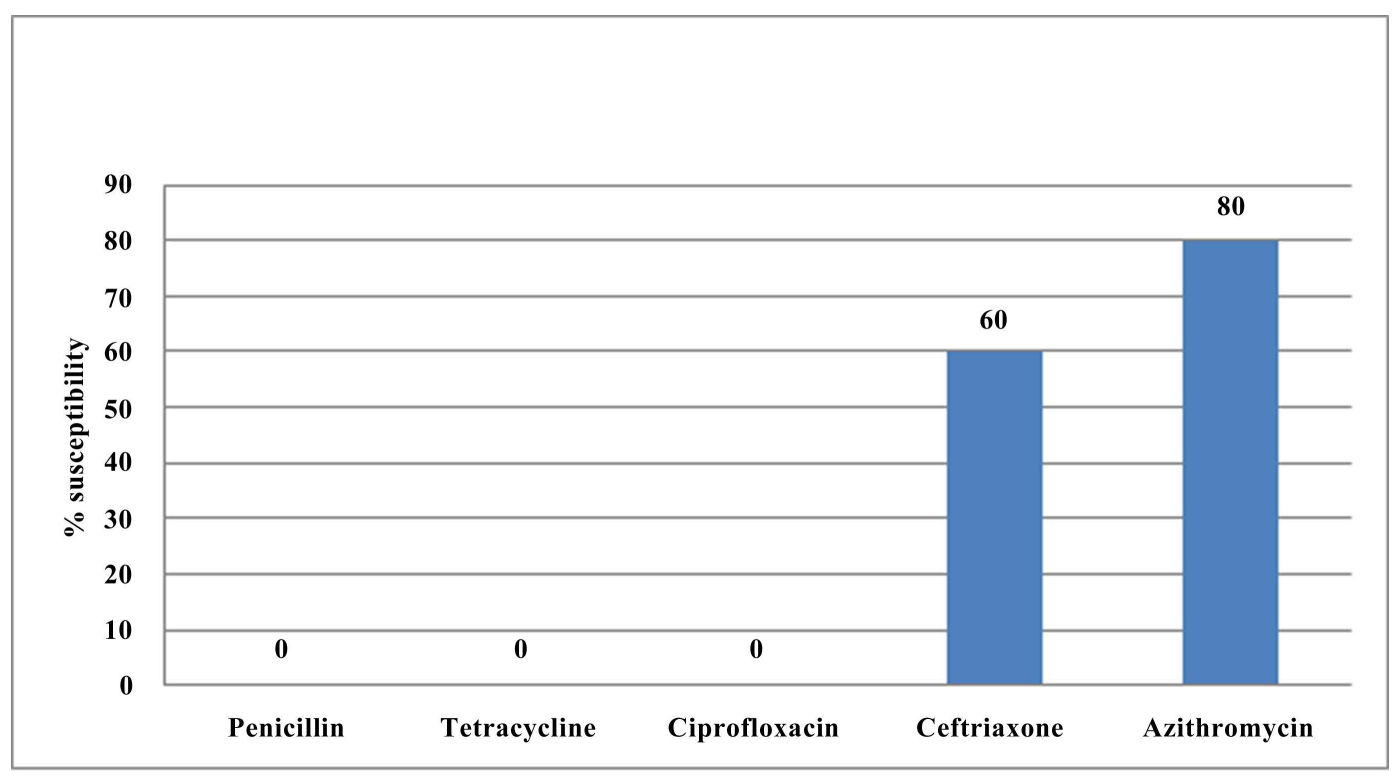

Figure 3. This chart shows percentage susceptibility of gonococcal isolates to different antibiotics. 


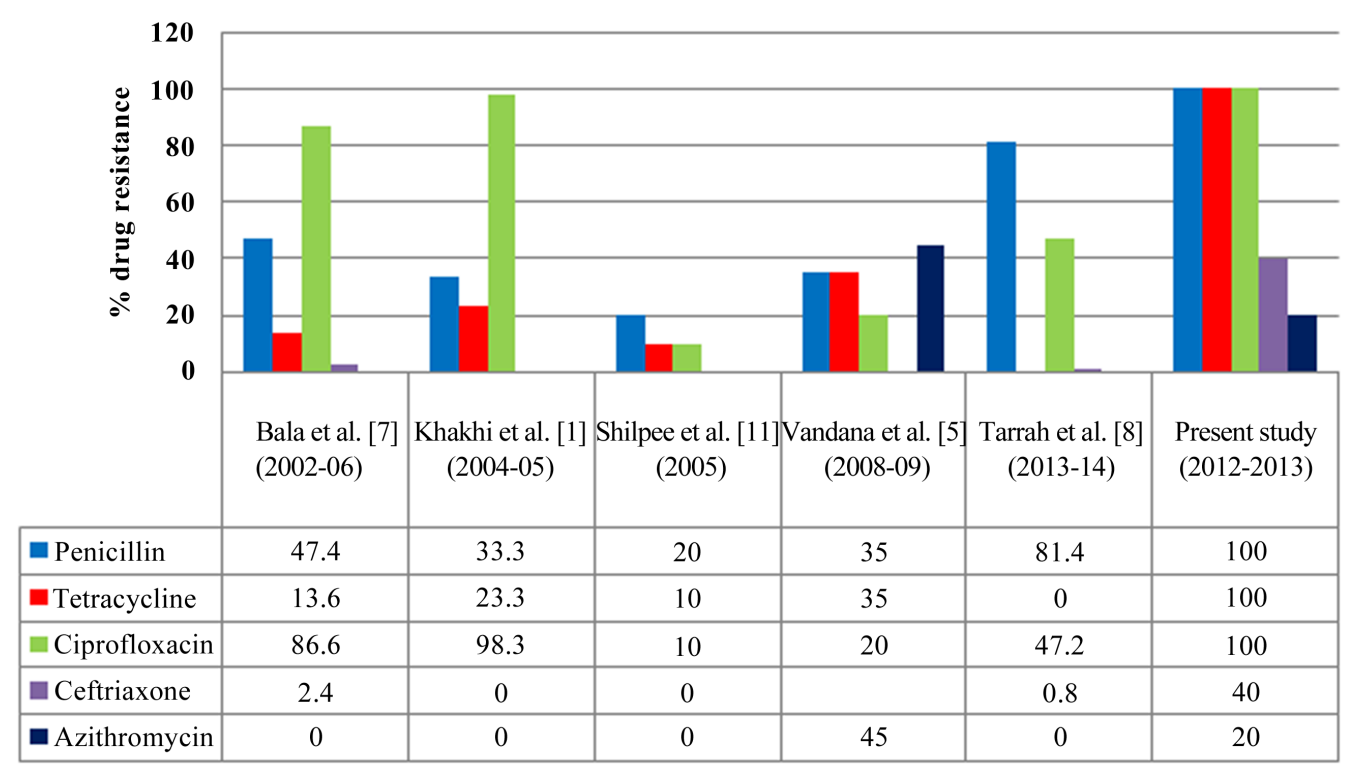

Figure 4. Comparision of proportion of drug resistance in $N$. gonorrhoeae with other India studies.

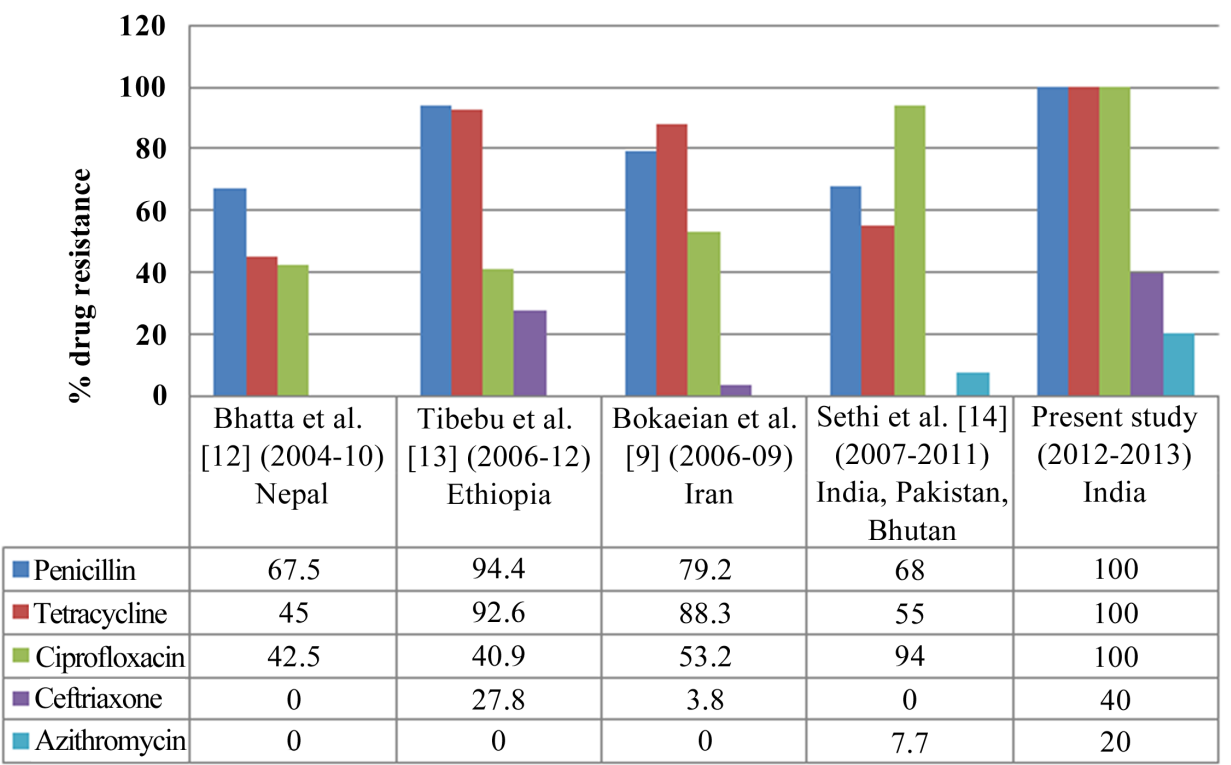

Figure 5. Comparision of proportion of drug resistance in $N$. gonorrhoeae with other countries studies.

Neisseria gonorrhoeae. In present study, 100\% resistance was recorded in penicillin, tetracycline and ciprofloxacin, while it was $40 \%$ in ceftriaxone and $20 \%$ in azithromycin. There is a gradual increase in resistance to penicillin, tetracycline and ciprofloxacin. Though resistance to third generation cephalosporin and azithromycin is low, it is increasing steadily.

Comparison of drug resistance in N. gonorrhoeae in India and other countries of world with present study is shown in Figure 4 \& Figure 5 respectively. Penicillin resistance is predominant everywhere while resistance to ciprofloxacin is more in Indian studies as compared to Nepal, Ethiopia and Iran, while that of tetracycline is almost similar in these countries. Though resistance to ceftriaxone and azithromycin is low, it is increasing with time [12]-[14].

Limitation of the present study is that there is less isolation rate of $N$. gonorrhoeae from female patients. Thus there is a need for better screening of asymptomatic patients, also for improving the isolation rate. Various drug resistance mechanisms like PPNG are not studied in detail so further analysis can be performed. 


\section{Conclusion}

Gonorrhoea is one of the classical sexually transmitted infections (STIs) and its complications are amongst the most frequent communicable diseases in many countries. It is essential to strengthen the diagnostic techniques, case reporting and epidemiological surveillance to find out asymptomatic and symptomatic cases of Neisseria gonorrhoeae. Clinicians need to consider treatment of gonorrhea based on individual susceptibility patterns instead of the routine empirical treatment of discharge syndromes. Surveillance for antimicrobial resistance is crucial for monitoring the emergence and spread of antibiotic resistance in gonococcal isolates. As noticed in present study also, there is development of resistance to third generation cephalosporin and azithromycin, drugs should be cautiously used.

\section{References}

[1] Khakhi, P., Bhalla, P., Sharma, P., Chawla, R. and Bhalla, K. (2007) Epidemiological Analysis of Neisseria gonorrhoeae Isolates by Antimicrobial Susceptibility Testing, Auxotyping and Serotyping. Indian Journal of Medical Microbiology, 25, 225-229.

[2] Bhalla, P., Bharara, T., Chakravarti, A., Garg, V.K., Rawat, D. and Sardana, K. (2015) Rising Trend of Antimicrobial Resistance among Neisseria gonorrhoeae Isolates and the Emergence of N. gonorrhoeae Isolate with Decreased Susceptibility to Ceftriaxone. Indian Journal of Medical Microbiology, 33, 39-42.

[3] Florence, P., Otim, F., Okongo, F., Ogwang, M. and Greco, D. (2012) The Prevalence and Antibiotics Susceptibility Pattern of Neisseria gonorrhoeae in Patients Attending OPD Clinics at St. Mary's Hospital Lacor Uganda. Journal of Preventive Medicine and Hygiene, 53, 186-189.

[4] Bhargava, D., Shakya, B., Mondal, K. and Rija, B. (2010) Emergence of Penicillin Resistant Neisseria gonorrhoeae. Journal of Institute of Medicine, 32, 15-18. http://dx.doi.org/10.3126/jiom.v32i1.3996

[5] Vandana, W.V. and Saxena, N.K. (2012) A Study of the Antimicrobial Susceptibility Pattern of Neisseria gonorrhoeae. Journal of Dental and Medical Science, 1, 1-4.

[6] Bala, M. (2011) Antimicrobial Resistance in Neisseria gonorrhoeae in South-East Asia. Regional Health Forum, 15, 63-73.

[7] Bala, M., Ray, K., Gupta, S.M., Muralidhar, S. and Jain, R.K. (2007) Changing Trends of Antimicrobial Susceptibility Patterns of Neisseria gonorrhoeae in India and the Emergence of Ceftriaxone Less Susceptible N. Gonorrhoeae Strains. Journal of Antimicrobial Chemotherapy, 60, 582-586. http://dx.doi.org/10.1093/jac/dkm238

[8] Tarrah, A., Kumar, S., Omran, A.N. and Tehrani, E.F. (2015) Investigation of Resistance to the Antibiotic Penicillin, Ceftriaxone, Ciprofloxacin and Cefixime in Neisseria gonorrhoeae Sampled from Patients Infected with the Gonorrhea in Poona City, India. Advanced Studies in Biology, 7, 11-16.

[9] Bokaeian, M., Qureshi, M.I., Dabiri, S. and Fard, A.H. (2011) An Investigation on Antibiotic Resistance of Neisseria gonorrhoeae Isolated from Gonorrheal Patients in Zahedan, Iran from 2007 to 2010. African Journal of Microbiology Research, 5, 2455-2459.

[10] Whiley, D.M., Limnios, E.A., Ray, S., Sloots, T.P. and Tapsall, J.W. (2007) Diversity of penA Alterations and Subtypes in Neisseria gonorrhoeae Strains from Sydney, Australia, That Are Less Susceptible to Ceftriaxone. Antimicrobial Agents and Chemotherapy, 51, 3111-3116. http://dx.doi.org/10.1128/AAC.00306-07

[11] Shilpee, C., Ramachandran, V.G., Das, S. and Bhattacharya, S.N. (2008) Antimicrobial Susceptibility Profile of Neisseria gonorrhoeae at STI Clinic. Indian Journal of Medical Microbiology, 26, 62-64. http://dx.doi.org/10.4103/0255-0857.38861

[12] Bhatta, D.R., Gokhale, S., Ansari, M.T., Tiwari, H.K., Gaur, A., Mathuria, J.P. and Ghosh, A.N. (2012) Gonococcal Infections: The Trends of Antimicrobial Susceptibility of Neisseria gonorrhoeae in Western Nepal. Nepal Journal of Medical Sciences, 1, 74-78. http://dx.doi.org/10.3126/njms.v1i2.6603

[13] Tibebu, M., Shibabaw, A., Medhin, G. and Kassu, A. (2013) Neisseria gonorrhoeae Non-Susceptible to Cephalosporins and Quinolones in Northwest Ethiopia. BMC Infectious Diseases, 13, 415. http://dx.doi.org/10.1186/1471-2334-13-415

[14] Sethi, S., Golparian, D., Bala, M., Dorji, D., Ibrahim, M., Jabeen, K. and Unemo, M. (2013) Antimicrobial Susceptibility and Genetic Characteristics of Neisseria gonorrhoeae Isolates from India, Pakistan and Bhutan in 2007-2011. BMC Infectious Diseases, 13, 35. http://dx.doi.org/10.1186/1471-2334-13-35 